\title{
Depth of Penetration in Gas Metal Arc Welding
}

\author{
P. E. Murray \\ Lockheed Martin Idaho Technologies Company \\ Idaho National Engineering and Environmental Laboratory \\ Idaho Falls, Idaho 83415-2210 \\ A. Scotti \\ Universidade Federal De Uberlandia \\ 38406-026, Uberlandia, Brazil

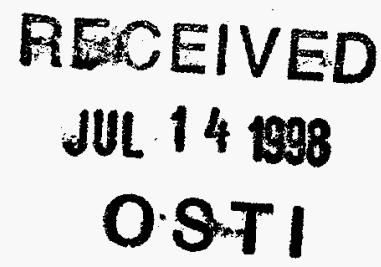

\begin{abstract}
We present a model of the depth of penetration in gas metal arc welding. This model is based on the assumption that the heat and mass transfer to the weld pool and the depth of penetration may be correlated by a dimensionless relation. This correlation leads to an analytical expression for depth of penetration, which involves empirical constants that are related to the efficiency of heat and mass transfer to the pool. We examine the accuracy of the model by comparing the theoretical depth of penetration and the measured depth of the weld pool for a range of processing variables encompassing short arc and free flight mass transfer. Measurements are obtained from bead on plate welds of stainless steel using a stainless steel electrode and a shielding gas that is rich in argon. The results confirm that the depth of penetration is affected by variations in the rate of mass transfer.
\end{abstract}

\section{Introduction}

Gas metal arc welding uses a consumable metal electrode which melts in the presence of the intense heat of the electric arc. Droplets are expelled from the electrode and impinge the base metal in order to reinforce the weld. Therefore energy transfer from the electric arc to the base metal is augmented by the energy of droplets impinging the pool. Transfer of droplets affects many aspects of the welding process including the size, shape and depth of penetration of the weld pool. Penetration is affected by a spray of droplets with sufficient momentum to carry energy deep into the pool and to stir the pool in order to enhance convective mixing. ${ }^{1}$ In some cases the momentum of droplets causes a deep, narrow penetration often seen in gas metal arc welding using a shielding gas that is rich in argon and contains a small amount of oxygen. ${ }^{2}$ In contrast, penetration in nonconsumable electrode processes such as gas tungsten arc welding is affected by flow in the weld pool caused by surface tension, electromagnetic and buoyancy forces. ${ }^{3}$

Theoretical models of heat transfer to the weld pool have been used to predict the size and shape of the cross sectional area of fused metal. Notable examples include the models due to Adams $^{4}$ and Christensen et al. ${ }^{5}$ which are based on Rosenthal's ${ }^{6}$ model of a moving point source of energy, and the extension to a moving distributed source of energy obtained by Eagar and Tsai. $^{7}$ These models assume steady state energy transfer to the base metal by conduction only, and the geometrical idealization of a planar base metal is used to obtain analytical solutions to the energy equation. In particular, the area of the weld pool depends on the heat flux into the base metal, the change in enthalpy that is needed to melt the base metal, the thermal diffusivity of the base metal, and the travel speed. However, the effects of convective heat transfer in the weld pool and the deposition of filler metal are not included. To overcome these limitations, numerical models of heat transfer have been recently developed. These include heat transfer models due to Tekriwal and Mazumder ${ }^{8}$ and Pardo and Weckman, ${ }^{9}$ and fluid flow models due to Tsao and $\mathrm{Wu},{ }^{10} \mathrm{Kim}$ and $\mathrm{Na},{ }^{11}$ and Ushio and Wu. ${ }^{12}$ In fact, good agreement between actual and simulated shapes of the weld pool was obtained in particular cases. However, the effect of deposition of filler metal was included by fixing the mass, velocity and rate of droplets impinging the pool rather than direct numerical simulation of the dynamics of droplets detaching from the electrode.

The experimental data reported by Essers and Walter ${ }^{1}$ suggests a relationship between the momentum of droplets impinging the pool and the depth of penetration. In particular, Essers and Walter ${ }^{1}$ correlated the momentum of droplets and the depth of penetration in order to explain the presence of a deep, narrow penetration that is often seen in gas metal arc welding. This suggests that the depth of penetration is affected by the mass, velocity and rate of droplets impinging the pool. The mass, velocity and rate of droplets expelled from the electrode are determined by the melting rate of the electrode and the electromagnetic, gravitational, inertial and drag forces acting on a droplet forming at the tip of the electrode. ${ }^{13}$. Moreover, the velocity of droplets impinging the pool is affected by the arc length and the acceleration caused by flow of gas in the arc. A recent trend in numerical studies of the weld pool had been to develop models that include the effects of heat transfer in the arc, mass transfer from the electrode, gas flow in the arc, heat transfer and fluid flow in the pool, and droplets impinging the pool. A model of gas metal arc welding in which all these effects have been fully coupled has not been developed. 


\section{DISCLAIMER}

This report was prepared as an account of work sponsored by an agency of the United States Government. Neither the United States Government nor any agency thereof, nor any of their employees, makes any warranty, express or implied, or assumes any legal liability or responsibility for the accuracy, completeness, or usefulness of any information, apparatus, product, or process disclosed, or represents that its use would not infringe privately owned rights. Reference herein to any specific commercial product, process, or service by trade name, trademark, manufacturer, or otherwise does not necessarily constitute or imply its endorsement, recommendation, or favoring by the United States Government or any agency thereof. The views and opinions of authors expressed herein do not necessarily state or reflect those of the United States Government or any agency thereof. 


\section{DISCLAIMER}

Portions of this document may be illegible electronic image products. Images are produced from the best available original document. 
Therefore, an empirical correlation between depth of penetration and heat and mass transfer to the weld pool may be the best approach for predicting the depth. Although an empirical correlation between depth of penetration and welding parameters has been known awhile, ${ }^{14}$ another empirical correlation was recently presented. ${ }^{15}$ However, these studies did not explicitly include the effect of droplets impinging the weld pool. Moreover, Essers' and Walter's ${ }^{1}$ correlation between the depth of penetration and the momentum of droplets may be applicable only if the droplets carry enough momentum and energy in order to penetrate the weld pool and carry energy to the base of the pool. This is more likely to occur in the case of free flight transfer and less likely to occur in the case of short arc transfer.

In this study we use experimental and theoretical methods to explore the manner in which mass transfer by droplets impinging the pool affects the depth of penetration in gas metal arc welding. This study is motivated by experimental data which clearly shows that a change in mass transfer at constant heat transfer affects the depth of penetration. In order to explain this effect, we develop a theoretical model of the depth of penetration of the weld pool which includes the effects of mass transfer of droplets impinging the weld pool and heat transfer from the arc. The rate of transfer of droplets, heat transfer from the arc, and depth of penetration are successfully correlated by a dimensionless relation which is used to predict the depth of the weld pool for a range of processing variables. The accuracy of the model is examined by comparing theoretical predictions and experimental measurements of the depth of the pool which are obtained from bead on plate welds of stainless steel in a shielding gas that is rich in argon. The results show that the theoretical depth of penetration is a suitable indicator of the depth of the weld pool in the cases of short arc and free flight mass transfer. The scatter in the experimental data is ascribed to uncertainty in the measurement of depth and variations in the arc length which accompany the transition from short arc to free flight transfer.

\section{Experiment}

Horizontal bead on plate welds were made on $1 / 4$ and $1 / 2$ inch thick stainless steel type ASTM 304 using an automated gas metal arc welding apparatus. A Miller Maxtron 450 power supply was used in the constant voltage mode. The shielding gas was a mixture consisting of $98 \% \mathrm{Ar}_{2}$ and $2 \% \mathrm{O}_{2}$ and the flow rate of gas was fixed at 35 standard $\mathrm{cfh}$. The electrodes were stainless steel type AWS ER308L with diameters equal to $0.89 \mathrm{~mm}$ and $1.14 \mathrm{~mm}$. An experiment was designed to vary all the important processing variables that affect the depth of penetration, which include voltage, current, rate of deposition, electrode size, travel speed, arc length and mode of mass transfer.

Twenty seven separate welds were made to produce a variation in voltage from $17 \mathrm{v}$ to $35 \mathrm{v}$ and a variation in current from $90 \mathrm{~A}$ to $390 \mathrm{~A}$, which encompassed short arc and free flight transfer of metal from the consumable electrode. The arc was filmed using a special camera designed for a high intensity light source. The mode of metal transfer and the frequency of droplets detaching from the electrode were recorded for each weld. The observed mode of mass transfer was either short arc or streaming. In case the transfer was short arc with intermittent streaming, the mode is called mixed. In some cases of streaming transfer, the droplets in the stream were indistinguishable, and in that case the frequency was set to the maximum resolution of the measurement which is approximately equal to 500 droplets per second.

The metallographic samples were prepared by electrochemical etching and examined using a microscope. Magnified photographs were obtained and the depth of the weld pool was measured using calipers. The depth is defined as the distance from the original surface of the base metal to the bottom of the pool of fused metal. Therefore the height of the weld bead was not included in the measured depth. Two samples were prepared for each experimental condition, in order to ascertain the uncertainty in the measurement. The uncertainty is defined as the standard deviation of the difference in depth between samples from the same experimental condition. An estimate of the standard deviation indicates that the uncertainty in the measurement is equal to $0.5 \mathrm{~mm}$. In some cases the weld pool reached the base of a $1 / 4$ inch thick plate, and the weld was repeated using a $1 / 2$ inch thick plate. Although a change in the size of the plate alters the heat transfer, the full penetration welds were used in order to include the variability due to the difference between heat transfer in thin and thick plate welds.

\section{Model}

The model is based on the assumption that the depth of the weld pool, the heat transfer from the arc, and the mass transfer due to droplets impinging the weld pool may be correlated by a dimensionless relation. Therefore, we introduce a characteristic thermal length scale, $\alpha / \mathrm{S}$, where $\alpha$ is the thermal diffusivity of the base metal and $S$ is the travel speed. We also introduce a characteristic length scale for mass transfer, which is the radius of droplets impinging the pool. Using these characteristic length scales, we define three important dimensionless variables. The dimensionless depth of penetration is

$$
\mathrm{D}=\frac{\mathrm{d}}{\alpha / \mathrm{s}} \text {, }
$$

where $d$ is the depth of penetration. The dimensionless mass transfer number is

$$
\mathrm{A}=\frac{\dot{\mathrm{M}}}{\mu \mathrm{r}} \text {, }
$$

where $\dot{M}$ is the melting rate of the electrode, $\mu$ is the viscosity of the pool, and $\mathrm{r}$ is the radius of droplets impinging the pool. The dimensionless heat transfer number is

$$
\mathrm{B}=\frac{\mathrm{VIS}}{\Delta \mathrm{H} \alpha^{2}} \text {, }
$$

where $\mathrm{V}$ is the arc voltage, $\mathrm{I}$ is the current, and $\Delta \mathrm{H}$ is the change in enthalpy of the base metal that is heated from the initial temperature to the melting temperature.

The dependence of melting rate on current and electrode extension may be obtained from the correlation due to Lesnewich. ${ }^{16}$ Although the current and electrode extension are measured in the experiment, the melting rate is obtained directly from the relation $\dot{M}=\rho \pi R^{2} U$, where $\rho$ is the electrode density, 
$R$ is the electrode radius, and $U$ is the speed of the electrode. The mass of a droplet detaching from the electrode is obtained from the following relation,

$\mathrm{m}=\frac{\dot{\mathrm{M}}}{\mathrm{v}}$,

where $m$ is the average mass and $v$ is the measured frequency of detaching droplets. Assuming a spherical droplet, the radius is related to the mass,

$\mathrm{m}=\rho \frac{4}{3} \pi \mathrm{r}^{3}$,

where $r$ is the radius of the droplet.

Previous attempts to correlate depth of penetration and processing variables frequently led to mathematical relations that involve exponents that are determined empirically.

However, the uncertainty in $\mathrm{D}$ may introduce a numerical error in the computation of the exponents. A more accurate computation may be obtained by defining the efficiency of penetration,

$$
\delta=\frac{\mathrm{d}}{\sqrt{\frac{\mathrm{VI}}{\Delta \mathrm{HS}}}}=\frac{\mathrm{D}}{\sqrt{\mathrm{B}}} .
$$

To establish a mathematical and physical bases for a correlation between $\mathrm{A}, \mathrm{B}$ and $\delta$, we consider a general relationship that is given by

$\delta=F\left(A B^{n}\right)$,

where $F\left(A B^{n}\right)$ is an arbitrary function of the variable $A B^{n}$ and $\mathrm{n}$ is a positive constant. This implies that $\delta$ satisfies the following equation,

$\mathrm{nA}\left(\frac{\partial \delta}{\partial \mathrm{A}}\right)_{\mathrm{B}}-\mathrm{B}\left(\frac{\partial \delta}{\partial \mathrm{B}}\right)_{\mathrm{A}}=0$.

Hence the exponent $\mathrm{n}$ satisfies the following relation,

$\mathrm{n}=\left(\frac{\partial(\log \delta)}{\partial(\log B)}\right)_{\mathrm{A}}\left(\frac{\partial(\log \mathrm{A})}{\partial(\log \delta)}\right)_{\mathrm{B}}$,

where the subscript denotes the variable that is held constant in the evaluation of the derivative. Experimental data on the variation in $\delta$ with respect to variations in $\mathrm{A}$ and $\mathrm{B}$ may be used to determine the exponent empirically. Using the experimental data shown in Figs. 2 and 3, we found from Eq. (9) that $n=1 / 2$. Hence $\delta$ is a function of $A B^{1 / 2}$. Furthermore, we found from linear regression that the correlation between $\mathrm{AB}^{1 / 2}$ and $\delta$ may be accurately represented by the following relationship,

$\delta=\eta\left(\mathrm{AB}^{1 / 2}\right)^{1 / 3}$,

where $\eta$ is a positive constant. Using Eq. (6) in Eq. (10), we obtain the final result that is given in dimensionless variables,

$\mathrm{D}=\eta \mathrm{A}^{1 / 3} \mathrm{~B}^{2 / 3}$.

The coefficient $\eta$ in the preceding equation is related to the variation in depth with respect to variations in mass and heat transfer to the weld pool, and so we examined the possibility that $\eta$ may depend on the mode of mass transfer. However, we found that setting $\eta$ equal to a constant, $\eta=0.024$, leads to an excellent correlation for the range of processing variables used in the experiment. In fact, using linear regression to determine $\eta$ for each mode of mass transfer leads to a negligible improvement in the accuracy of the correlation.

Using the definitions of A, B and D in Eq. (11), we obtain the final result that is given in dimensional variables,

$\mathrm{d}=0.024\left(\frac{\rho \pi \mathrm{R}^{2} \mathrm{U}}{\mu \mathrm{r}}\right)^{1 / 3}\left(\frac{\mathrm{VI}}{\Delta \mathrm{H} \alpha}\right)^{2 / 3}\left(\frac{\alpha}{\mathrm{S}}\right)^{1 / 3}$.

The depth of penetration given by Eq. (12) is a function of material properties and processing variables that are directly controlled or easily measured in an experiment. However, measurements of the size and rate of droplets impinging the pool requires special instruments to view the flight of droplets in the arc. This is not a shortcoming, for a reliable estimate of the radius of droplets may be obtained from models of metal transfer. ${ }^{17} \mathrm{~A}$ final note is the properties of steel which are given by $\rho=8000 \mathrm{~kg} / \mathrm{m}^{3}, \alpha=7.510^{-6} \mathrm{~m}^{2} / \mathrm{s}, \Delta \mathrm{H}=1.010^{10} \mathrm{~J} / \mathrm{m}^{3}$, and $\mu=4.010^{-3} \mathrm{~kg} / \mathrm{m} \mathrm{s}$.

\section{Results}

Although heat transfer has the largest effect on the depth of penetration, mass transfer has a significant effect too. This observation is confirmed by inspecting actual profiles of the weld pool which are shown in Fig. 1. We observe that the depth of penetration is affected by variations in the mass transfer number at constant heat transfer number. In particular, a comparison of Figs. $1 \mathrm{a}$ and $1 \mathrm{~b}$ shows that an increase in depth may be explained by an increase in the rate of mass transfer. A comparison of Figs. 1c and 1d leads to the same conclusion. However, a change in mass transfer at constant heat transfer may be accompanied by changes in current, voltage, arc length, mode of transfer, and the momentum and energy of droplets impinging the weld pool. Therefore, the depth of penetration may be affected by several physical variables that are related in a complex process. This underscores the importance of using dimensionless variables in order to simplify the analysis and reduce the number of variables that are needed to describe the depth of penetration.

The relationship between the mass transfer number, heat transfer number, and efficiency of penetration is shown in Figs. 2 and 3 . In particular, we observe that increasing the mass transfer number at constant heat transfer number leads to an increase in the efficiency of penetration. In a similar manner, increasing the heat transfer number at constant mass transfer number leads to an increase in the efficiency of penetration. These results are noteworthy, for they suggest the need to include the effect of mass transfer in a model of depth of penetration and provide a sound basis for a correlation between $A, B$ and $\delta$. In the case that the derivatives $(\partial(\log \delta) / \partial(\log B))_{\mathrm{A}}$ and $(\partial(\log \delta) / \partial(\log \mathrm{A}))_{\mathrm{B}}$ are exact constants, the general solution to Eq. (8) is given by Eq. (7). Although the uncertainty in depth precludes an accurate approximation of the derivatives, we found that $(\partial(\log \delta) / \partial(\log B))_{\mathrm{A}} \cong 0.4$ and $(\partial(\log \delta) / \partial(\log A))_{B} \cong 0.8$. The computed derivatives were used 
in Eq. (9) in order to deduce that the exponent in Eq. (7) is equal to $1 / 2$. Hence a mathematical and physical bases for a correlation between $\mathrm{AB}^{1 / 2}$ and $\delta$ has been established. Furthermore, computation of the depth of penetration is facilitated by using linear regression to obtain a simple and fitting mathematical relationship between $A B^{1 / 2}$ and $\delta$, which is given by Eq. (10). The final result is a linear correlation between $\mathrm{A}^{1 / 3} \mathrm{~B}^{2 / 3}$ and $\mathrm{D}$, which is given by Eq. (11).

The correlation between the measured dimensionless depth of the weld pool and the heat and mass transfer numbers given by $A^{1 / 3} B^{2 / 3}$ is shown in Fig. 4. This correlation is an accurate model of the relationship between heat transfer, mass transfer and depth of penetration. To underscore this point, statistical measures of the accuracy of the model were obtained from linear regression. We found that the adjusted correlation coefficient is equal to 0.955 , the value of the $F$ test from analysis of variance is equal to 555 , and the standard error of regression is equal to 0.498 . These results confirm the accuracy of the correlation between $A^{1 / 3} B^{2 / 3}$ and $D$. In particular, a large value of the correlation coefficient and a small value of the standard error indicate that the correlation is meaningful and accurate. Moreover, a large value of the $\mathrm{F}$ test indicates that the variability in $D$, which is due to the influence of the variables $A$ and $B$, is large in comparison to the uncertainty in $\mathrm{D}$. Therefore the correlation is also reliable.

The correlation between the measured depth of the weld pool and the theoretical depth of penetration obtained from Eq. (12) is shown in Fig. 5. These results show some scatter which may be ascribed to uncertainty in the measurement of depth and variations in the mode of mass transfer. In fact, the mode of mass transfer and the arc length are strongly correlated, and the transition from short arc transfer to streaming transfer is accompanied by a change in arc length. According to the results shown in Fig. 5, the largest scatter occurs in the mixed mode of mass transfer, in which the variation in arc length is the largest. We propose that these variations affect the efficiency of heat and mass transfer to the pool. Notwithstanding these variations, the standard error in the correlation between measured and theoretical depths is approximately equal to $0.5 \mathrm{~mm}$. Since the standard error may be ascribed to the uncertainty in the experimental measurement, we conclude that the theoretical depth of penetration is a suitable indicator of the depth of the pool in the range of processing variables used in the experiment. In contrast, the theoretical depth obtained from considering heat transfer or mass transfer only is not always a suitable indicator of the depth of the pool, as is confirmed by the results shown in Fig. 1. Another noteworthy observation is that a change from short arc transfer to streaming transfer is accompanied by an increase in depth which is largely due to an increase in the heat transfer number and partly due to an increase in the mass transfer number.

\section{Summary}

We developed a model that correlates depth of penetration and mass and heat transfer to the weld pool in gas metal arc welding. This model led to an analytical expression for depth of penetration, which is given by Eq. (12) and involves empirical constants that are related to the efficiency of mass and heat transfer to the weld pool. Experimental data on the variation in depth with respect to variations in heat and mass transfer numbers were used to establish a mathematical and physical bases for the correlation given by Eq. (12). This correlation is unique, for it includes the effects of heat transfer from the arc and mass transfer due to droplets impinging the weld pool.

The model was used to predict the depth of the weld pool for a range of welding process variables encompassing variations in voltage, current, travel speed, electrode size, rate of deposition of filler metal, arc length and mode of mass transfer. We examined the accuracy of the model by comparing theoretical predictions and experimental measurements of the depth of the weld pool which were obtained from bead on plate welds of stainless steel using a shielding gas that is rich in argon. The results obtained in this study confirm that the depth of penetration is affected by the rate of mass transfer. Moreover, we found that the theoretical depth of penetration is a suitable indicator of the measured depth of the weld pool in the cases of short arc transfer, streaming transfer and the transition from short arc to free flight transfer. Further studies will be undertaken to ensure that the model may be used to correlate heat and mass transfer to the weld pool and the depth of the weld pool for other welding materials and shielding gases.

\section{Acknowledgments}

Work supported by the U. S. Department of Energy, Office of Basic Energy Sciences under DOE Idaho Operations Contract No. DE-AC07-94ID13223. The authors are grateful to Dr. H. B. Smartt for suggesting this study.

\section{References}

1. W. G. Essers and R. Walter, Welding J., 60, 37s-42s (1981).

2. V. R. Dillenbeck and L. Castagno, Welding J., 66, 45s-49s (1987).

3. K. C. Mills and B. J. Keene, Int. Mater. Rev., 35, 185-216 (1990).

4. C. M. Adams, Welding J., 37, 210s-215s (1958).

5. N. Christensen, V. de L. Davies, and K. Gjermundsen, British Welding J., 2, 54-75 (1965).

6. D. Rosenthal, Trans. ASME, 68, 849-866 (1946).

7. T. W. Eagar and N.-S. Tsai, Welding J., 62, 346s-355s (1983).

8. P. Tekriwal and J. Mazumder, Welding J., 67, 150s-156s (1988).

9. E. Pardo and D. C. Weckman, Metall. Trans. B, 20,937947 (1989).

10. K. C. Tsao and C. S. Wu, Welding J., 67, 70s-75s (1988).

11. J.-W. Kim and S.-J. Na, Welding J., 74, 141s-152s (1995).

12. M. Ushio and C. S. Wu, Metall. Trans. B, 28, 509-516 (1997).

13. J. H. Waszink and L. H. J. Graat, Welding J., 62, 109s-116s (1983).

14. C. E. Jackson and A. E. Shrubsall, Welding J., 32, 172s-178s (1953).

15. I. S. Kim, A. Basu, and E. Siores, Int. J. Adv. Manuf. Technol., 12, 393-401 (1996).

16. A. Lesnewich, Welding $J ., 37,343 \mathrm{~s}-353 \mathrm{~s}$ (1958).

17. Y.-S. Kim and T. W. Eagar, Welding J., 72, 269s-278s (1993).

18. A. Lesnewich, Welding J., 37, 418s-425s (1958). 


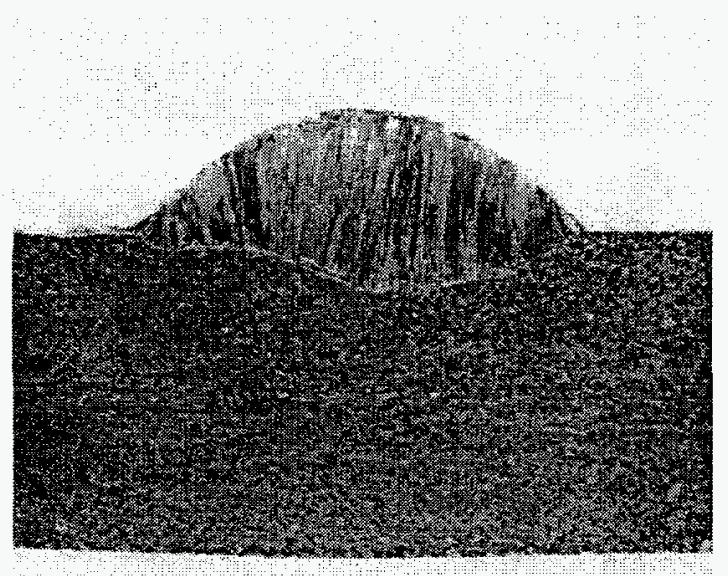

Fig. 1a. Depth $=1.4 \mathrm{~mm}, A=100, B=25, D=0.75$.

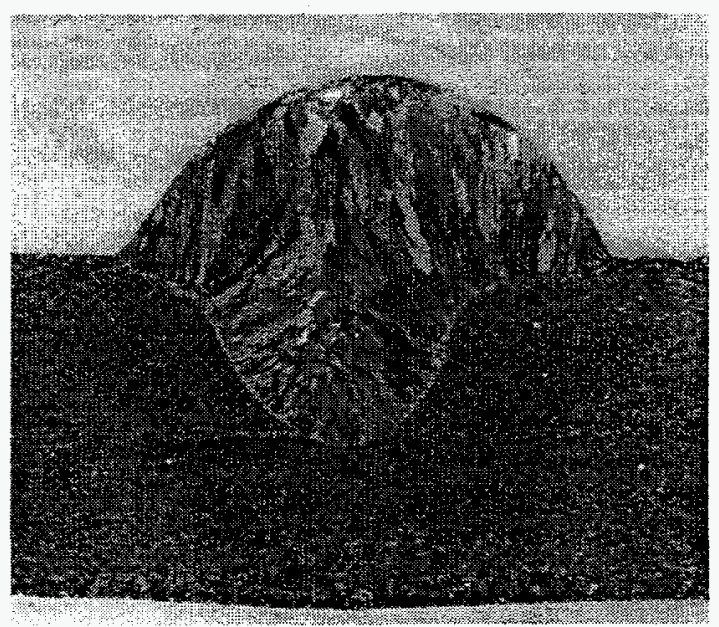

Fig. 1b. Depth $=3.7 \mathrm{~mm}, \mathrm{~A}=310, \mathrm{~B}=25, \mathrm{D}=2.0$.

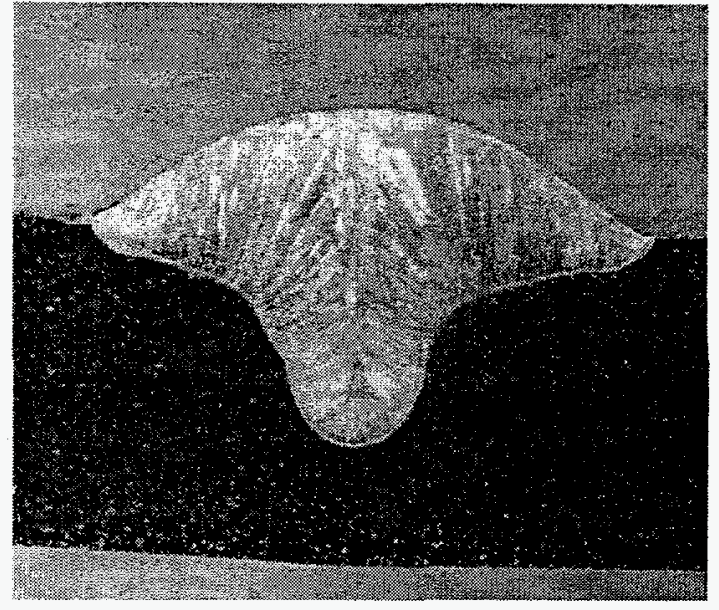

Fig. 1c. Depth $=4.1 \mathrm{~mm}, \mathrm{~A}=720, \mathrm{~B}=160, \mathrm{D}=5.5$.

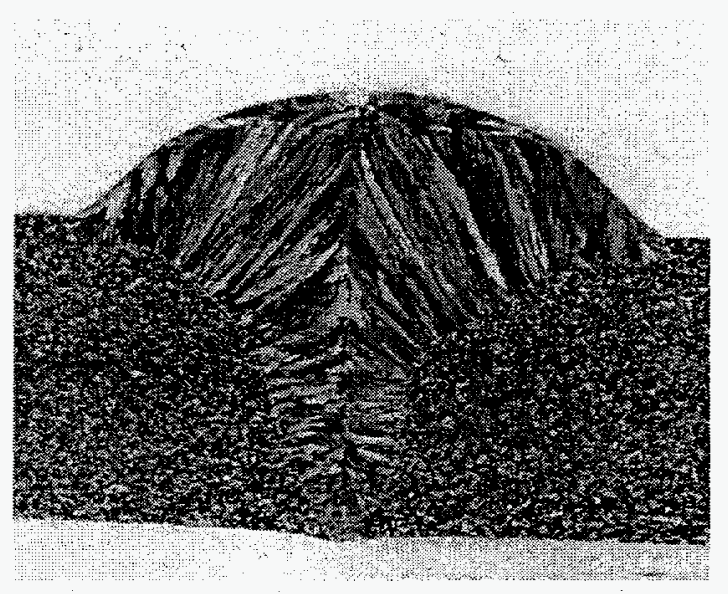

Fig. 1d. Depth $=5.7 \mathrm{~mm}, \mathrm{~A}=870, \mathrm{~B}=160, \mathrm{D}=7.6$.

Fig. 1. Profiles of the weld pool for various values of the heat and mass transfer numbers. The depth of penetration is affected by variations in the mass transfer number at constant heat transfer number. 


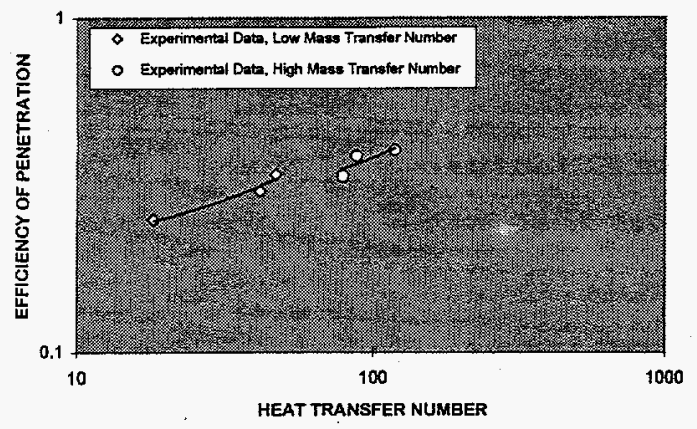

Fig. 2. Correlation between the efficiency of penetration and the heat transfer number at constant values of the mass transfer number. The slope of the correlation $(\partial(\log \delta) / \partial(\log B))_{\mathrm{A}} \cong 0.4$.

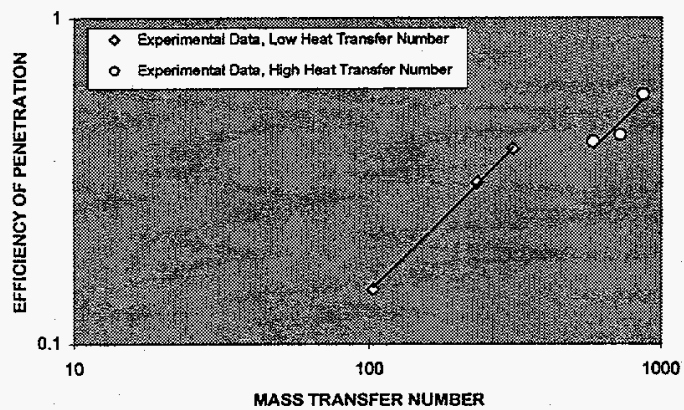

Fig. 3. Correlation between the efficiency of penetration and the mass transfer number at constant values of the heat transfer number. The slope of the correlation $(\partial(\log \delta) / \partial(\log A))_{B} \cong 0.8$.

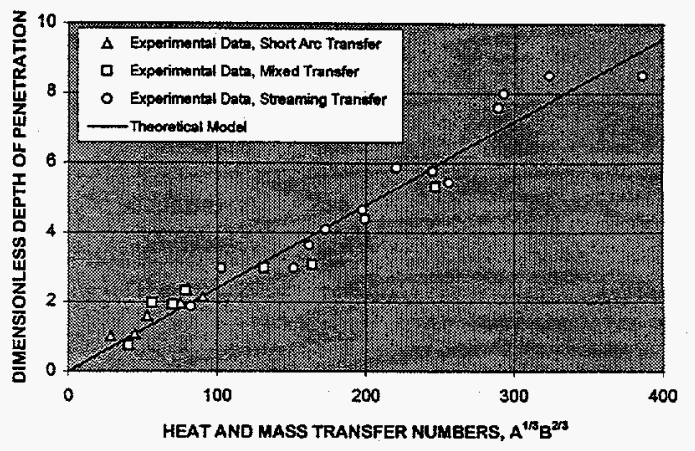

Fig. 4. Correlation between the measured depth of the weld pool and the heat and mass transfer numbers, $\mathrm{A}^{1 / 3} \mathrm{~B}^{2 / 3}$. The correlation is linear, passes through the origin, and has a slope equal to 0.024 .

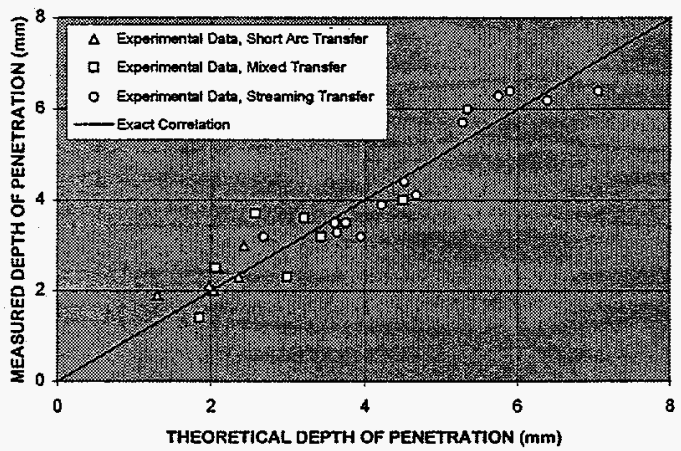

Fig. 5. Correlation between the measured depth of the weld pool and theoretical depth of penetration from Eq. (12). The accuracy of the correlation is affected by the mode of mass transfer and the uncertainty in measurement. 